\title{
Inverted Left atrial appendage after Left ventricular assist device implantation
}

\author{
Roshith Chandran ${ }^{1}$ and Vikrant Pathania ${ }^{1}$ \\ ${ }^{1}$ Freeman Hospital
}

September 21, 2020

\begin{abstract}
Left atrial appendage inversion is a rare event in cardiac surgery and can often be mistaken for a mass, thrombus or vegetation. This can be a challenge especially in case of a left ventricular assist device (LVAD) where a correct diagnosis is of crucial significance. Intra-operative TEE, is an invaluable tool to detect an inverted left atrial appendage and differentiate it from other potentially dangerous diagnosis like left atrial clot. Knowledge of this entity along with a high index of suspicion can help early diagnosis of this scenario and avoid unnecessary reinstitution of cardiopulmonary bypass and re-surgery
\end{abstract}

\section{Keywords:}

Left atrial mass; Left Ventricular Assist Device; Trans-Esophageal Echocardiography

\section{Introduction:}

Post cardiac surgery left atrial appendage inversion is a rare event and can cause diagnostic dilemma, as it can be mistaken for a mass, thrombus or vegetation. This can be a challenge in case of a left ventricular assist device (LVAD) where a correct diagnosis is of crucial significance.

\section{Case report:}

A previously fit and well 29 years old female, presented to our hospital with gradually progressive shortness of breath and pedal oedema, after giving birth to a child 2 months ago. 2D Echo revealed an ejection fraction less than $10 \%$ with dilated ventricles. A diagnosis of peripartum cardiomyopathy was made and she was commenced on heart failure medications. Patient had poor response to medical management and suffered a cardiac arrest in the hospital. Post successful resuscitation, it was decided to consider her for heart transplant. Considering the COVID epidemic and paucity of available donors, it was decided to support her mechanically with a left ventricular assist device (LVAD). After an uneventful LVAD (HVAD, HeartWare, Medtronics Inc, Minnesota MN, USA) implantation via midline sternotomy and standard cardiopulmonary bypass, device was activated and the revolutions on the pump were steadily increased to 2600 RPM for de-airing on cardiopulmonary bypass. The power on the LVAD device increased to a maximum of 7.5 Watts and the flows dropped. As the de-airing proceeded a suspicious hyper-echoic mass was noted in the left atrium on TEE leading to confusion of a LA clot. Inversion of left atrial appendage was suspected since the pre-CPB trans-esophageal echocardiogram showed no LA clot and it was unlikely that a new clot would form in an anticoagulated patient on CPB. The LVAD flows were reduced and re-examination of the left atrium by the surgeon revealed an inverted appendage, which was promptly everted with help of a forceps. LA mass disappeared, LVAD flows stabilised and the power utilization immediately dropped to 3.2 Watts. The patient made an uneventful recovery and was discharged from the hospital within 2 weeks of surgery.

\section{Discussion:}


Discovery of a left atrial mass intraoperatively raises a suspicion of a clot, tumor or vegetation. Suction force produced by left ventricular assist device, can cause deformity of the left atrial appendage causing inversion. In case of severe heart failure, LVAD is activated and flows are gradually increased as the cardio-pulmonary bypass flows are reduced, in a bid to transition from full CPB to assisted flows with the LVAD. Increased suction force from the LVAD, especially in presence of low blood volume in the left ventricular chamber can pull blood from the left atria across the mitral valve and can result in inversion of the left atrial appendage. It is also possible that the appendage may invert during the de-airing manoeuvres11Bouzas-Mosquera A, Alvarez-García N, Cuenca-Castillo JJ. Inverted left atrial appendage. Heart 2008;94:1064.. On TEE, it may present as a "crooked finger" or with a tongue-like appearance22Leong MC, Latiff HA, Hew CC, Mazlan SL, Osman H. Inverted left atrial appendage masquerading as a cardiac mass. Echocardiography 2013;30:E335.. Apart from causing confusion in the diagnosis, it can cause mitral valve obstruction and impaired ventricular filling33Molaei A, Tabib A, Meraji M, Shemirani RH. Inverted left atrial appendage: A cause of left ventricularinflow obstruction. Iran Cardiovasc Res J 2010;4:139-41. and necrosis and rupture of the left atrial wall. It can also lead to distortion of the pulmonary veins causing pulmonary congestion and subsequent right ventricular failure, especially in cases of borderline right ventricular function44Gattani R, Ekanem E, Shea J, Zhao Q, Singh R, Liam RP. Left atrial appendage inversion presenting as acute right ventricular failure after left ventricular assist device implantation. Journal of the American College of Cardiology. 2020 Mar;75(11):2389. Patients with dilated cardiomyopathy have functional mitral regurgitation and subsequent LA dilation with enlarged appendage and thus are susceptible to appendage inversion. Inability to identify the mass as an inverted LAA can result in unnecessary return to CPB, surgical intervention, and additional ischemia time. Regular pre-operative trans-esophageal echocardiographic assessment for patients undergoing LVAD implantation includes assessment of left atrial appendage for clots, especially in presence of severe MR and atrial fibrillation55Flores AS, Essandoh M, Yerington GC, Bhatt AM, Iyer MH, Perez W, et al. Echocardiographic assessment for ventricular assist device placement. Journal of Thoracic Disease. 2015 Dec 17;7(12):2139-50.. It is essential to have a high index of suspicion for an inverted LAA, especially when a new mass appears post-surgery. Comparison with pre-operative images will help clinch the diagnosis especially with the knowledge that the mass is homogenous and arises from the anterolateral wall of the atrium just superior to the mitral valve and inferior to the pulmonary veins. Absence of left atrial appendage when seen in the mid-esophageal two chamber view and mid esophageal left atrial appendage view along with typical shape of the mass with a broad base and highly mobile tip is highly suspicious of appendage inversion66Leong MC, Latiff HA, Hew CC, Mazlan SL, Osman H. Inverted left atrial appendage masquerading as a cardiac mass. Echocardiography. 2013 Feb;30(2):E33-5.. Inverted appendage generally everts spontaneously with fluid loading of LV (Trendelenberg manoeuvre or Valsalva manoeuvre) or by transiently reducing LVAD flows. Other methods to correct it include digital manipulation, or using forceps77Chikwe J, Fischer GW, Adams DH. Inverted left atrial appendage. J Am Coll Cardiol 2009;54:e7.. Rarely, LA appendage ligation might be required to prevent re-inversion.

Intra-operative TEE is thus an invaluable tool to detect an inverted LAA and differentiate it from other potentially dangerous diagnosis like left atrial clot, which would have mandated unnecessary reinstitution of cardiopulmonary bypass and re-surgery.

List of Images:

Figure 1: Mid Esophageal 4 chamber view with color doppler across the mitral valve showing the inverted left atrial appendage causing flow turbulence

Figure 2: Mid Esophageal 4 chamber view showing the mass, with the LVAD

Figure 3: Mid Esophageal 2 chamber view showing the typical appearance of the inverted left atrial appendage

References: 

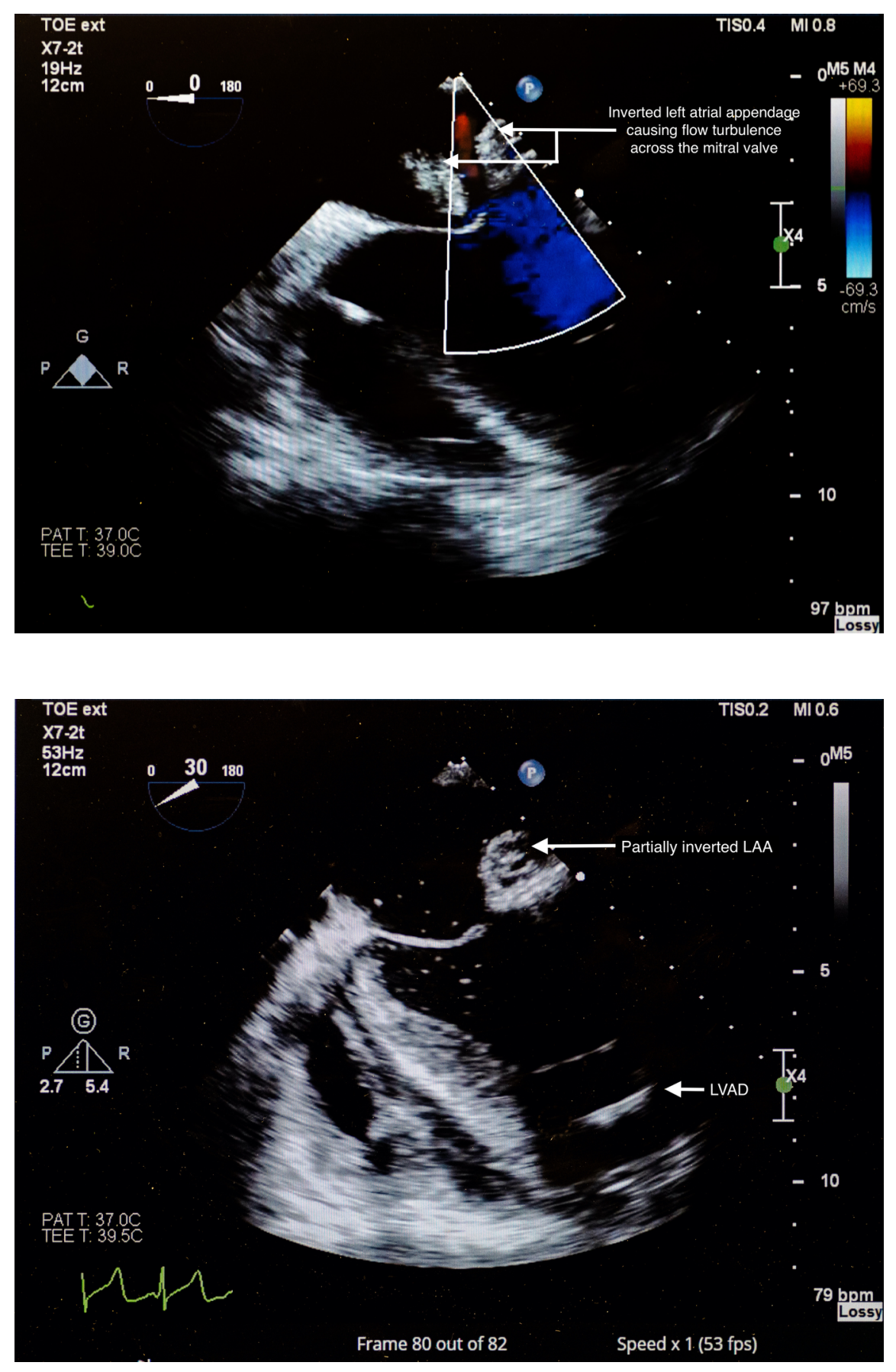


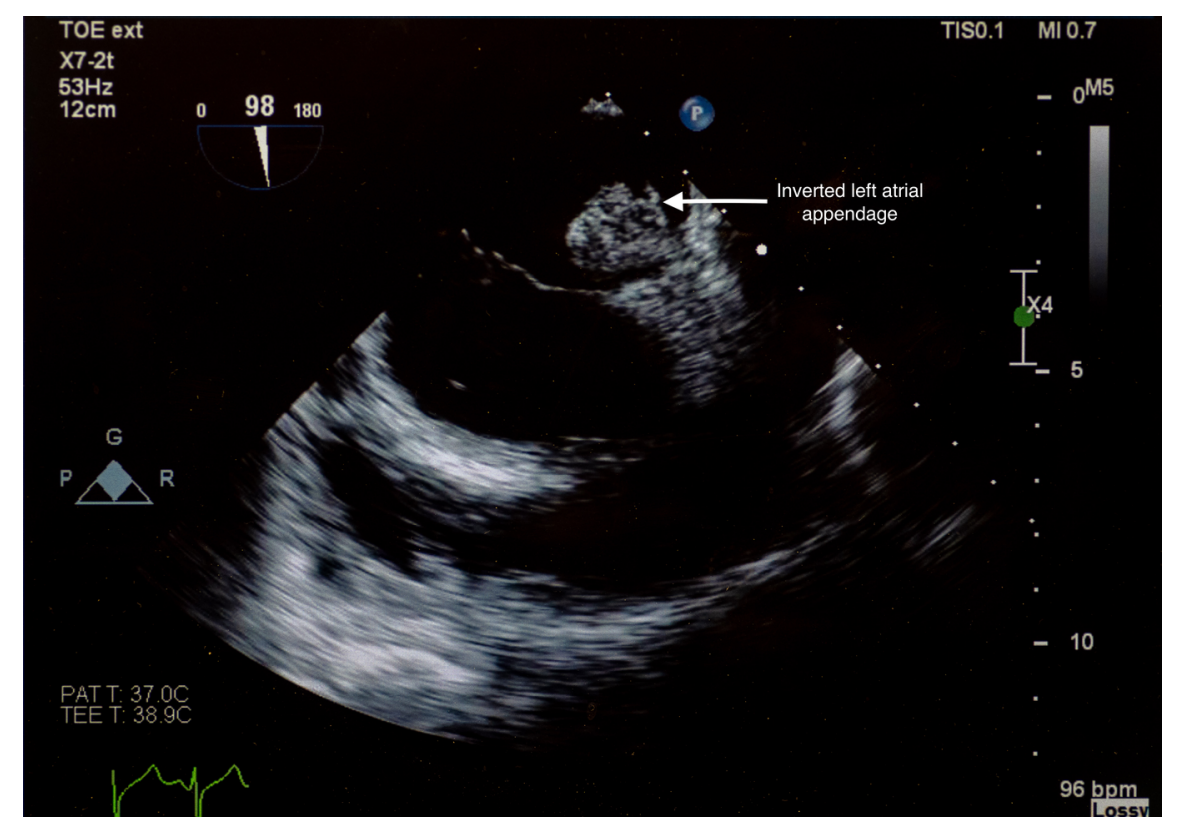

